\title{
Endoscopic ultrasound-guided gastrojejunostomy with a lumen-apposing metal stent: a multicenter, international experience
}

Authors

Institutions
Amy Tyberg ${ }^{1}$, Manuel Perez-Miranda ${ }^{2}$, Ramon Sanchez-Ocaña ${ }^{2}$, Irene Peñas ${ }^{2}$, Carlos de la Serna ${ }^{2}$, Janak Shah ${ }^{3}$, Kenneth Binmoeller ${ }^{3}$, Monica Gaidhane' ${ }^{1}$, lan Grimm ${ }^{4}$, Todd Baron ${ }^{4}$, Michel Kahaleh ${ }^{1}$

Institutions are listed at the end of article. submitted

17. November 2015

accepted after revision

15. December 2015

\section{Bibliography}

DOI http://dx.doi.org/

10.1055/s-0042-101789

Endoscopy International Open 2016; 04: E276-E281

(c) Georg Thieme Verlag KG

Stuttgart · New York

E-ISSN 2196-9736

\section{Corresponding author}

\section{Michel Kahaleh, MD, FASGE}

Division of Gastroenterology and Hepatology

Weill Cornell Medical College New York, NY 10021

USA

Fax: +1-646-962-0110

mkahaleh@gmail.com

\section{License terms}

Background: Surgical gastrojejunostomy and enteral self-expanding metal stents are efficacious for the management of gastric outlet obstruction but limited by high complication rates and shortterm efficacy. Endoscopic ultrasound-guided gastrojejunostomy (EUS-GJ) is a novel alternative option.

Patients and methods: Patients who underwent EUS-GJ between March 2014 and September 2015 as part of a prospective multicenter registry at four academic centers in two countries were included. Technical success was defined as successful placement of a gastrojejunal lumen-apposing metal stent. Clinical success was defined as the ability of the patient to tolerate an oral diet. Postprocedural adverse events were recorded.

Results: The study included 26 patients, of whom 11 (42\%) were male. Technical success was

\section{Introduction}

$\nabla$

Surgical gastrojejunostomy is the preferred treatment for gastric outlet obstruction (GOO), but it carries a complication risk of up to $40 \%$ [1]. Endoscopic placement of an enteral self-expanding metal stent (SEMS) is an alternative treatment option, with technical success rates between $90 \%$ and $100 \%$ and clinical success rates between $67 \%$ and $100 \%$. In comparison with surgery, enteral SEMS placement is associated with lower complication rates, decreased time to the initiation of oral feeding, and shorter hospital stays [2-4]. However, stenting carries an increased need for reintervention, with patency rates as low as $57 \%$ after 6 months [4]. Additionally, a SEMS is not a good long-term solution for GOO with a benign cause.

Recently, the fully endoscopic creation of a gastrojejunal bypass (endoscopic ultrasound-guided gastrojejunostomy, or EUS-GJ) has emerged as a therapeutic option. This procedure involves locating the jejunum endosonographically from inside achieved in 24 patients (92\%). Clinical success was achieved in 22 patients (85\%). Of the 4 patients in whom clinical success was not achieved, 2 had persistent nausea and vomiting despite a patent EUS-GJ and required enteral feeding for nutrition, 1 died before the initiation of an oral diet, and 1 underwent surgery for suspected perforation. Adverse events, including peritonitis, bleeding, and surgery, occurred in 3 patients (11.5\%).

Conclusion: EUS-GJ is an emerging procedure that has efficacy and safety comparable with those of current therapies and should hold a place as a new minimally invasive option for patients with gastric outlet obstruction.

Clinical trial identification number:

NCT01522573

the stomach and placing a dedicated biflanged lumen-apposing metal stent (LAMS) across a newly formed fistulous tract. EUS-GJ has been validated in animal models [5-8], several case reports [9$12]$, and two case series to date $[13,14]$. We present an international, multicenter experience that aimed to further assess the efficacy and safety of EUS-GJ in patients having GOO with both benign and malignant causes.

\section{Methods}

$\nabla$

\section{Study overview}

All patients who underwent EUS-GJ between March 2014 and September 2015at four academic centers in two countries were included, except for four patients from one center who had previously been reported in a separate study [14]. Demographic data, procedural information, and clinical follow-up were captured. Technical success was defined as the successful placement of a gastrojejunal LAMS. Clinical success was de- 
Table 1 Characteristics and clinical data of 26 patients who underwent gastrojejunal bypass for the management of gastric outlet obstruction.

\begin{tabular}{|c|c|}
\hline Patients, $\mathrm{n}$ & 26 \\
\hline Sex, M/F & $11 / 15$ \\
\hline Mean age, $y$ & 66.2 \\
\hline \multicolumn{2}{|l|}{ Indication for EUS-G] } \\
\hline Malignant obstruction & 17 \\
\hline Benign obstruction & 9 \\
\hline \multicolumn{2}{|l|}{ Methods used to locate jejunal loop } \\
\hline \multicolumn{2}{|l|}{ Assisted methods } \\
\hline Contrast-filled balloon & 13 \\
\hline Ultra-slim scope & 5 \\
\hline Nasobiliary drain & 3 \\
\hline \multicolumn{2}{|l|}{ Unassisted methods } \\
\hline Direct EUS puncture & 3 \\
\hline NOTES & 2 \\
\hline Technical success & $24(92 \%)$ \\
\hline \multicolumn{2}{|l|}{ Failures and dropouts } \\
\hline Misplaced stents, successfully bridged & 5 \\
\hline Proximal flange: FCSEMS & 3 \\
\hline Distal flange: LAMS via NOTES & 2 \\
\hline $\begin{array}{l}\text { Misplaced stents, salvage not attempted and LAMS } \\
\text { removed }\end{array}$ & 2 \\
\hline Over-the-scope clip closure & 1 \\
\hline Intraluminal enteral SEMS & 1 \\
\hline $\begin{array}{l}\text { Clinical success } \\
\text { Failures and dropouts }\end{array}$ & $22(85 \%)$ \\
\hline Persistent nausea/vomiting requiring enteral feeding & 2 \\
\hline Death before introduction of oral diet & 1 \\
\hline $\begin{array}{l}\text { Surgical bypass at discretion of surgeon } \\
\text { despite successful EUS-G] }\end{array}$ & 1 \\
\hline Major adverse events & $3(11.5 \%)$ \\
\hline Peritonitis & 1 \\
\hline Bleeding & 1 \\
\hline Abdominal pain resulting in laparotomy & 1 \\
\hline
\end{tabular}

M, male; F, female; EUS-G], endoscopic ultrasound-guided gastrojejunostomy; NOTES, natural orifice transluminal endoscopic surgery; FC, fully covered selfexpanding metal stent; LAMS, lumen-apposing metal stent.

fined as the ability of the patient to tolerate an oral diet following the procedure. Adverse events included bleeding, stent migration, perforation, infection, and death. Multivariate logistic regression analysis was conducted to evaluate for any positive predictors for technical success and clinical success. Odds ratios were obtained for predictor variables such as patient demographics, medical history, concurrent medical conditions, and type of LAMS placed. Multivariate logistic regression analysis was conducted with SAS 9.3 (SAS Institute, Cary, North Carolina, USA).

\section{Procedure technique}

All procedures were performed by operators experienced in therapeutic EUS and LAMS placement while the patients were under general anesthesia or endoscopist-directed propofol sedation. An Axios LAMS (Boston Scientific; Marlborough, Massachusetts, USA) with or without a cautery tip was used in all cases. Several different approaches were used to identify the targeted jejunal loop: intraluminal canalization of the stricture (assisted approach) or direct determination of the location of the jejunal loop (unassisted approach) ( $\bullet$ Table 1 ).

\section{Balloon or nasobiliary drain catheter}

An endoscope was advanced into the stomach. A guidewire was coiled under fluoroscopic guidance into the jejunum past the stricture, under direct endoscopic view whenever feasible [10].

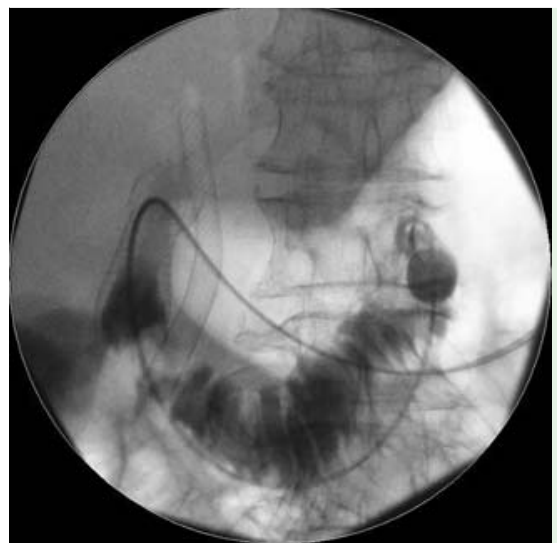

Fig. 1 Fluoroscopic visualization of a biliary extraction balloon inflated within the jejunum.

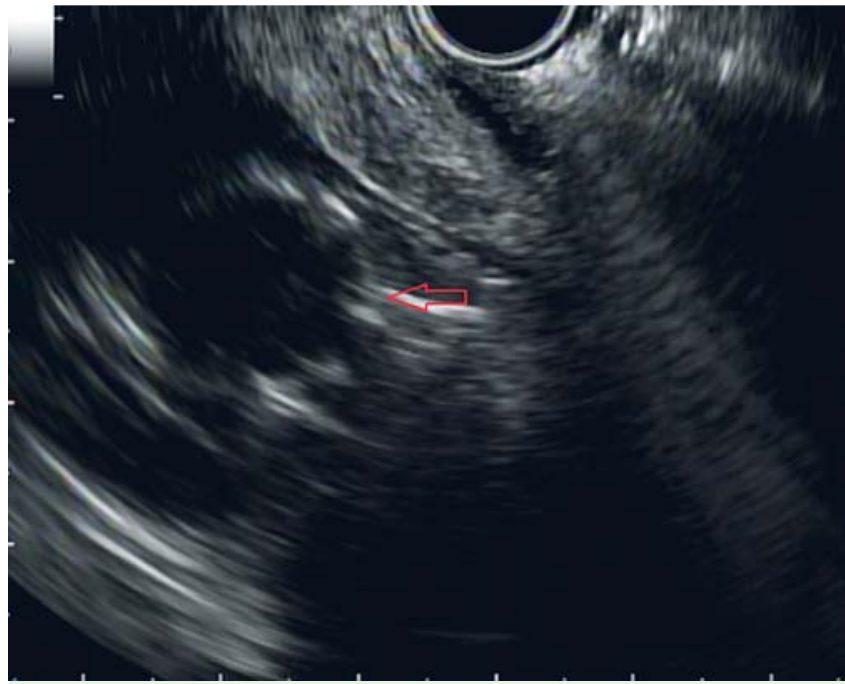

Fig.2 Endosonographic view of the inflated biliary extraction balloon.

If the stenosis could not be traversed with the endoscope, the wire was advanced from the stomach through the stenosis under fluoroscopic guidance alone. A dilating balloon, biliary extraction balloon ( Fig. 1), or nasobiliary drain catheter was advanced over the wire into the jejunum under fluoroscopic guidance. Balloon catheters required endoscope removal before insertion, whereas nasobiliary drain catheters were advanced through the endoscope, which was subsequently removed. Then, a linear echoendoscope was advanced into the stomach alongside the balloon or catheter. Contrast was injected into the balloon or directly into the jejunal lumen through the nasobiliary drain to help identify the jejunal loop echosonographically ( $\bullet$ Fig. 2 ) and fluoroscopically. The loop was then accessed with a 19-gauge needle, and a second guidewire was advanced through it into the jejunal lumen for over-the-wire LAMS insertion ( $\bullet$ Fig.3). If the cautery-tipped LAMS delivery system was not available, the fistulous tract was serially dilated with a needle-knife and a balloon. Conversely, if the cautery-tipped LAMS was available, the delivery system was inserted in one step without prior dilation, either over the wire or freehand, as previously described [13]. A LAMS with either a 10 - or 15 -mm diameter was deployed, and the balloon was dilated to its diameter ( $\bullet$ Fig.4, $\bullet$ Fig.5). 
Visualization with an ultra-slim scope (hybrid rendezvous) An ultra-slim endoscope was advanced either through a previously placed gastrostomy site or perorally into the stomach and then past the stricture into the jejunum. Water was injected through the ultra-slim scope to distend the bowel lumen. A linear echoendoscope was then advanced into the stomach (alongside the ultra-slim scope in cases in which the ultra-slim scope had been introduced perorally). The distended jejunal loop containing the ultra-slim scope was identified endosonographically and accessed with a 19-gauge EUS needle. Endoscopic visualization of the needle with the ultra-slim scope confirmed location within the bowel $(\bullet$ Video 1$)$. A guidewire was advanced through the needle and coiled within the bowel lumen. A forceps through the ultra-slim scope was used to grasp the guidewire for traction ( $\bullet$ Fig. 6). The fistulous tract was created and the LAMS deployed as previously described.

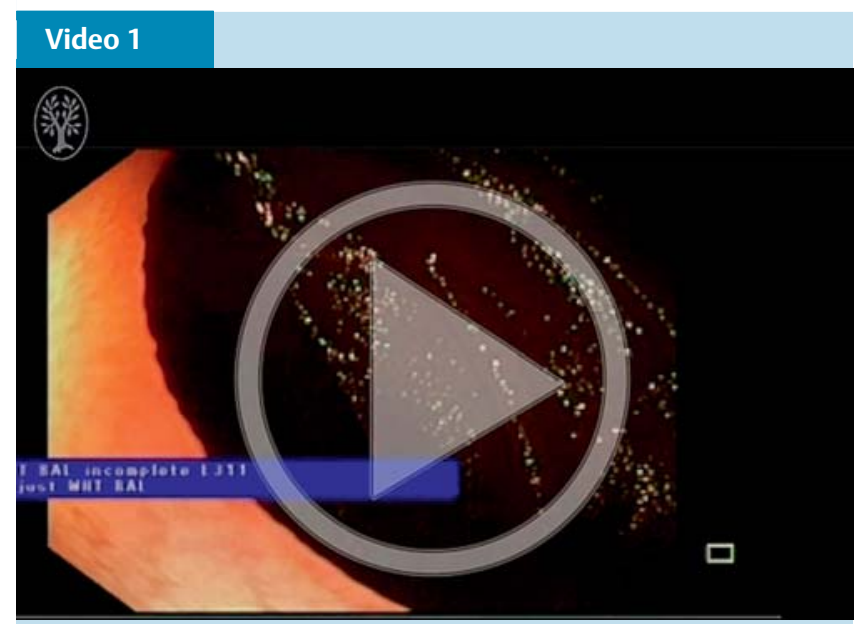

Endoscopic ultrasound-guided gastrojejunostomy created by using a hybrid rendezvous approach with an ultra-slim endoscope through a percutaneous endoscopic gastrojejunostomy track. Online content including video sequences viewable at: http://dx.doi.org/10.1055/s-0042-101789

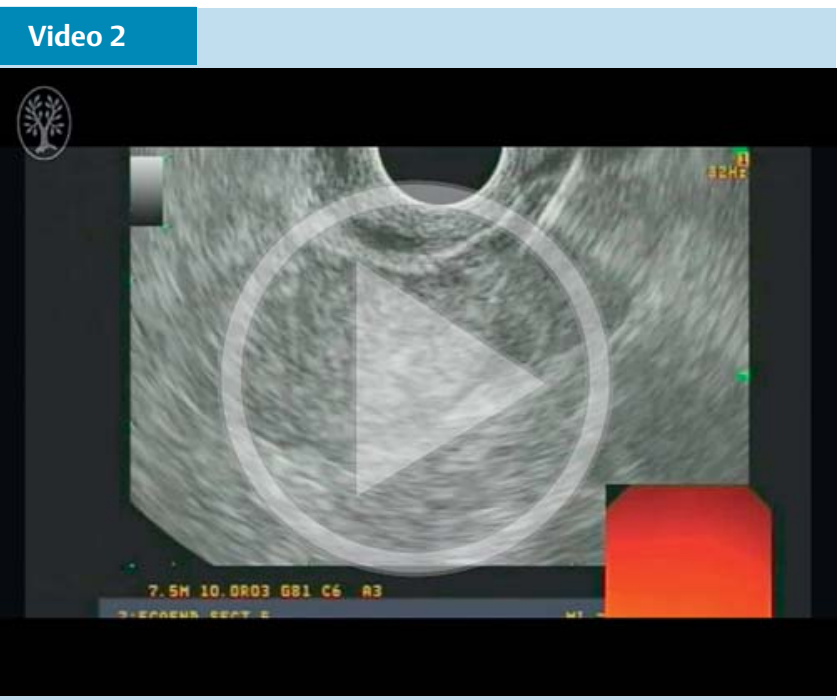

Endoscopic ultrasound-guided gastrojejunostomy created by using a 22gauge needle for saline injection and jejunal distension before puncture with a 19-gauge needle. Online content including video sequences viewable at: http://dx.doi.org/10.1055/s-0042-101789

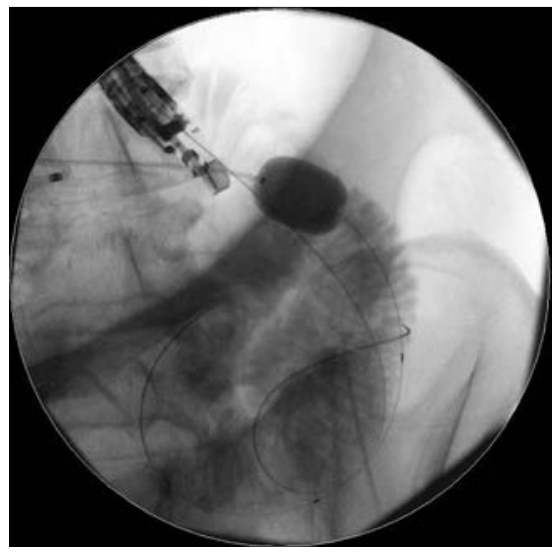

Fig. 3 Coiled guidewire within the targeted jejunal loop.

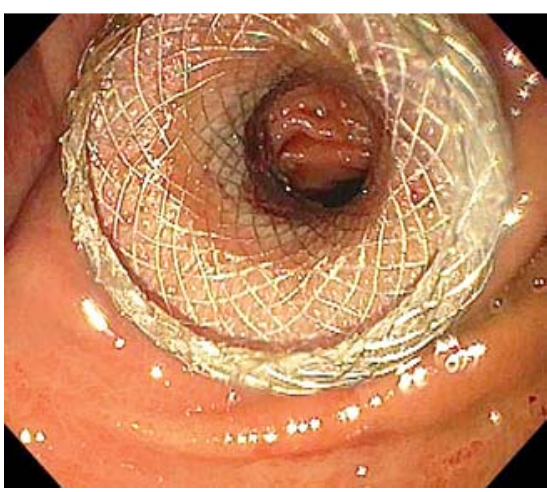

Fig. 4 Endoscopic view of a deployed gastrojejunal lumenapposing metal stent.

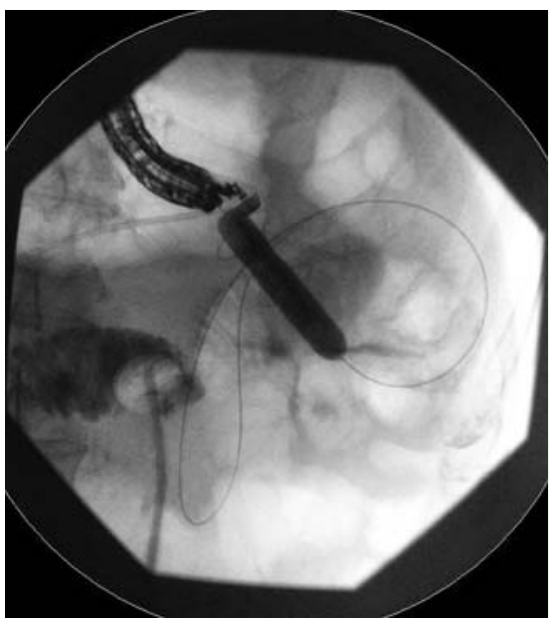

Fig. 5 Dilation of a deployed lumen-apposing metal stent to its diameter.

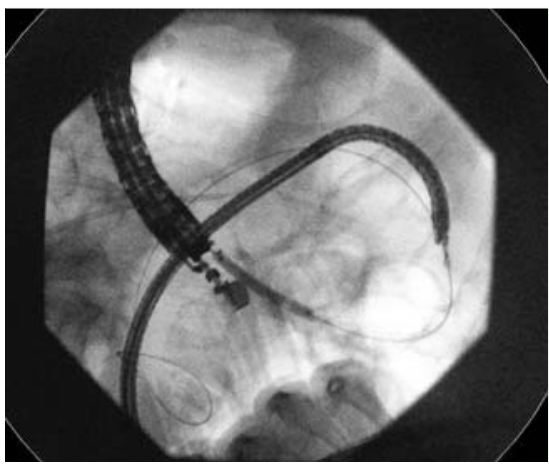

Fig. 6 Coiled guidewire in the targeted jejunal loop, grasped by forceps. through an ultra-slim scope in the jejunum. 


\section{Direct endoscopic ultrasound puncture}

A linear echoendoscope was advanced into the stomach. A jejunal loop was identified endosonographically and accessed with a 19gauge needle. If distended jejunal loops could not be imaged, a 22-gauge needle puncture was first made to distend the loops with saline before puncture with a 19-gauge needle ( $\bullet$ Fig. 7, - Video 2). Contrast was injected through a 19-gauge needle to confirm location within the small bowel. The fistulous tract was then created and the LAMS deployed as previously described.

\section{Natural orifice transluminal endoscopic surgery}

A linear echoendoscope was advanced into the stomach, and 19gauge needle access into the peritoneum was obtained close to the ligament of Treitz as previously reported [9]. A guidewire was passed through the needle into the peritoneal cavity and the echoendoscope removed, with the guidewire left in place. A double-channel gastroscope was advanced over the wire into the stomach, and a needle-knife incision was made on the gastric wall. The incision was balloon-dilated to $15 \mathrm{~mm}$ and the peritoneal cavity entered with the gastroscope. A jejunal loop was incised with a needle-knife under endoscopic view, and a guidewire was coiled within its lumen. A LAMS was advanced over the wire through the incision on the bowel wall, and its distal flange was deployed inside the jejunum. The gastroscope was then pulled back into the stomach, with both the opened LAMS flange and a rat-tooth forceps through the working channels [9] used as retractors. The proximal LAMS flange was then deployed inside the stomach, and a balloon was used to dilate the LAMS to its diameter.

\section{Results}

$\nabla$

A total of 26 patients were included in the study, of whom 11 were male $(42 \%)$ and whose mean age was 66.2 years (range 34-90). Indications for EUS-GJ included malignant $(n=17)$ and benign ( $n=9)$ GOO. The etiology of benign GOO was either chronic pancreatitis or pyloric stenosis. A total of 13 patients had undergone previous therapy with enteral SEMS placement, 3 had undergone dilation, and 5 had undergone placement of a percutaneous endoscopic gastrojejunostomy (PEG-J) tube or nasojejunal tube for feeding. Of the 26 patients, 8 (31\%) had anatomy altered by prior surgery, including Whipple procedure $(n=3)$, subtotal gastrectomy $(n=1)$, gastrojejunal bypass $(n=1)$, Roux-en-Y bypass $(n=2)$, and Billroth I procedure $(n=1)$. The mean postprocedural hospital stay was 5.7 days (range $0-34$ ).

\section{Technical success}

Technical success was achieved in 24 patients (92\%). The jejunal loop was located with an assisted method in 21 patients: balloon catheter $(n=13)$, ultra-slim scope $(n=5$; through a percutaneous tract in 3 cases and perorally in 2 cases), or nasobiliary drain catheter $(n=3)$. The jejunal loop was located with an unassisted method in 5 patients: direct EUS puncture $(n=3)$ or natural orifice transluminal endoscopic surgery (NOTES; $n=2$ ). All 3 patients who underwent direct EUS puncture required saline injection with a 22-gauge needle for luminal distention before jejunography with a 19-gauge needle. In 25 patients (96\%), a 15-mmdiameter LAMS was used, and in 1 patient, a 10-mm-diameter LAMS was placed. A cautery-tipped LAMS (Hot Axios; Boston Scientific) was used in 9 patients (35\%). In 7 patients, partial LAMS misplacement occurred, with either the proximal or distal flange

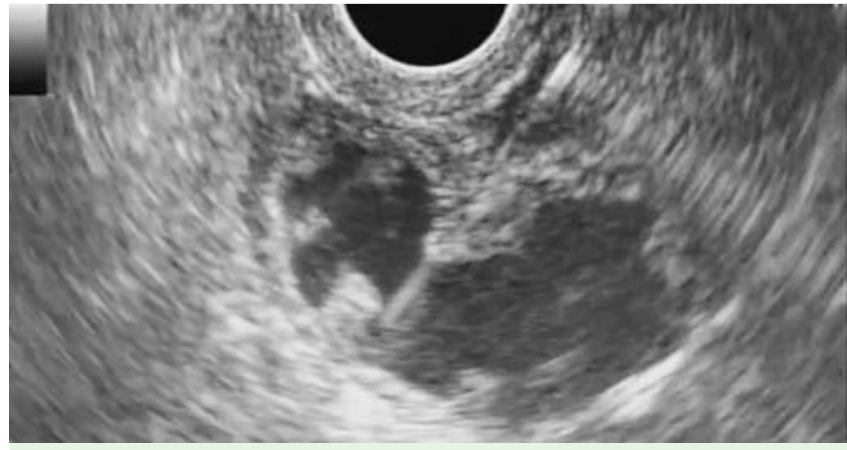

Fig. 7 Direct endoscopic ultrasound puncture of a water-distended jejunal loop.

slipping out of the targeted lumen. In all 3 patients with misplacement of the proximal flange beyond the gastric wall, the tract was successfully bridged with a telescoping fully covered SEMS (FCSEMS) as previously reported [12]. In 2 of the 4 patients with distal flange misplacement, the tract was salvaged with NOTES access ( 1 planned and 1 unplanned) and placement of a bridging LAMS instead of an FCSEMS. In the 2 patients with unsalvaged distal flange misplacement, the LAMS was pulled back into the stomach as previously reported [13], and the access site was closed with an over-the-scope clip $(n=1)$ or an enteral SEMS without any attempt at closure $(n=1)$. In 2 additional patients, a bridging FCSEMS was placed despite correct placement of a LAMS because of concerns for delayed migration arising from tenting of the LAMS after deployment. After adjustments for age, gender, etiology, presence or absence of previous intervention, presence or absence of altered anatomy, and use of LAMS with or without cautery for fistula creation, logistic regression analysis showed no significant predictors for technical success ( $P>0.8$ for all).

\section{Clinical success}

Clinical success was achieved in 22 patients (85\%): in 15 of the 17 with malignant GOO (88\%) and in 7 of the 9 with benign GOO (78\%). Of the 4 patients in whom clinical success was not achieved, 2 had persistent post-procedural nausea and vomiting despite a patent EUS-GJ and required enteral feeding for nutrition, 1 died before initiation of an oral diet, and 1 was taken for surgery because of abdominal pain following the procedure and underwent surgical bypass at the discretion of the surgeon despite evidence of a successful EUS-GJ with a bridged FCSEMS. Of the 5 patients who required placement of a bridging FCSEMS, clinical success was achieved in 4. Follow-up data were available for 19 patients. After a mean follow-up of 7.9 weeks (range $0-$ 32 ), no symptom recurrence was noted in any of the clinically successful cases.

Clinical success was achieved in 18 of the 19 patients who had undergone previous interventions (95\%) and in 6 of the 7 patients who had not undergone previous interventions (86\%). Clinical success was achieved in 7 of the 8 patients with altered anatomy (88\%). After adjustments for age, gender, etiology, presence or absence of previous intervention, presence or absence of altered anatomy, and use of LAMS with or without cautery, logistic regression analysis showed no significant predictors for clinical success $(P>0.9$ for all). 


\section{Adverse events}

Adverse events occurred in 3 patients (11.5\%). Post-procedural abdominal pain developed in 1 patient, who was taken to surgery before consultation with the endoscopist because of free air seen on imaging. Surgical evaluation revealed a correctly deployed LAMS with a bridging FCSEMS, but surgical gastrojejunostomy was still performed at the discretion of the surgeon. Peritonitis developed in 1 of the 2 patients with distal flange misplacement, whose LAMS had been withdrawn after insertion across the gastric wall despite closure with an over-the-scope clip. This patient had long-standing malignancy with ascites and peritoneal carcinomatosis and died the following day. In the other patient with failed LAMS placement, a standard uncovered enteral SEMS was placed through the luminal stricture without fistula closure. Post-procedural bleeding requiring transfusion developed, but no peritonitis or perforation, and this patient died of disease progression 4 months later. After successful LAMS placement, no migration or dysfunction was noted. After adjustments for age, gender, etiology, presence or absence of previous intervention, presence or absence of altered anatomy, and use of LAMS with or without cautery for fistula creation, logistic regression analysis showed no significant risk factors for immediate adverse events $(P>0.1$ for all). Of the 3 subjects who experienced immediate adverse events, all had undergone previous intervention, 2 were male (66\%), and 2 had altered anatomy (66\%).

\section{Discussion}

EUS-GJ is an emerging procedure that allows symptom relief in patients who have GOO without the risks of surgical bypass and the limited long-term efficacy of enteral SEMS placement [4]. The endoscopic technique avoids the wound-healing complications of a surgical operation while preserving the concept of a functional anastomosis away from the obstructed area. The covering of the LAMS can prevent the restenosis and subsequent symptom recurrence encountered with an uncovered enteral SEMS while avoiding the risk for migration associated with a covered enteral SEMS [13].

The feasibility of LAMS gastrojejunostomy first emerged in the literature in animal studies that used either EUS-based $[5,6]$ or NOTES-based approaches $[7,8]$. Subsequently, in several additional case reports [9-12] and two case series [13,14], EUS-GJ appeared safe and efficacious in humans. Our multicenter study is the largest experience to date, incorporating novel techniques to help locate the target bowel, a variant of the LAMS with cautery, and salvage strategies for LAMS misplacement. Additionally, our study included patients with both benign and malignant forms of GOO.

Locating the jejunum endosonographically can be challenging because it is difficult to know exactly which region of the bowel is being imaged. Secure location of the target is critical for EUS-GJ because loops of large and small bowel can look similar. Intraluminal assisted methods, such as standard $[10,14]$ or dedicated prototype $[6,11,13]$ balloons inflated with contrast or saline, have been used. However, balloon catheters require scope removal before insertion, potentially resulting in difficult peroral insertion across the stricture [14]. We used nasobiliary drain catheters as a novel alternative to balloon catheters for target location. Another novel assisted method for target location introduced in our series was the hybrid rendezvous (conducted perorally or percutaneously via PEG-J tracks) with ultra-slim endo- scopes ( Video 1). We also modified currently known unassisted methods of jejunal access by using a 22-gauge needle for saline injection and jejunal distension before puncture with a 19gauge needle ( Video 2 ). In our study, the individual endoscopists at each center employed a variety of techniques, highlighting different ways to overcome the greater challenges of EUS-GJ compared with EUS-guided LAMS insertion into less mobile targets, such as pancreatic pseudocysts. No significant correlation between technical or clinical success and the technique used was noted.

In addition to target identification, proper LAMS placement is another challenging step of EUS-GJ. The use of bridging FCSEMS to salvage partial LAMS misplacement was originally reported during EUS-guided gallbladder drainage. De la Serna-Higuera et al. used bridging FCSEMS in 4 of their 11 technically successful gallbladder cases (36\%) [15]. In our series, misplacement occurred in 7 of 26 cases (27\%) and was the only reason for technical failure overall. Proximal flange misplacement was salvaged with a bridging FCSEMS in all of our 3 patients in whom this occurred, as reported in detail elsewhere [12]. Itoi et al. encountered distal flange misplacement in 2 of 20 EUS-GJ cases, and this was the only reason for technical failure overall in their series [13]. We salvaged 2 of our 4 distal flange misplacements by using NOTES access and LAMS-in-LAMS bridging. In 1 of the 2 patients in whom this salvage strategy was undertaken, NOTES had been used from the outset [9], whereas in the other patient, conversion to NOTES from standard EUS-GJ was required.

Clinical success was achieved in 22 patients ( $85 \%$ ), which is an efficacy rate comparable with those of other techniques [2-4]. This success rate is significant because 19 patients in our series (73\%) underwent EUS-GJ after prior failed attempts at surgical or endoscopic therapy for GOO. Adverse events included 1 case of bleeding and 1 death in a severely debilitated patient, possibly due to procedure-related peritonitis. A third patient underwent surgery because of abdominal pain and a finding of free air on imaging before any discussion with the endoscopist. Surgical evaluation revealed a correctly placed stent. This emphasizes the need for excellent communication between disciplines. No other significant adverse events were encountered.

Limitations of this study include the retrospective design and lack of a control arm for comparison with other standard surgical or endoscopic therapies. Of note, different techniques for locating the jejunal limb echosonographically were employed, and several different variations of the LAMS were used at the different centers. However, the collective experience of excellent efficacy with minimal adverse events in 26 patients argues for the continued use of this procedure as a new minimally invasive option for patients with GOO. Additionally, several of the patients included in the study were severely ill and thus contraindicated for surgical bypass. EUS-GJ can offer palliation in patients too ill to undergo surgery. It can also be offered to patients with benign disease.

EUS-GJ with LAMS will ideally be more clinically successful than the same procedure with traditional enteral stents because enteral stents are inevitably affected by tumor ingrowth and tissue hyperplasia, whereas LAMS are placed away from the area of the cancer and are fully covered. And finally, in a health care system scrutinizing rising expenses, EUS-GJ appears to be a less expensive option than its surgical alternative.

In conclusion, EUS-GJ with placement of a LAMS is an emerging procedure having efficacy and safety comparable with those of current standard therapies and should hold a place as a new 
minimally invasive option for patients with luminal gastrointestinal obstruction.

Competing interests: Dr. Michel Kahaleh has received grant support from Boston Scientific, Fujinon, EMcison, Xlumena, W. L. Gore \& Associates, Mauna Kea Technologies, Apollo Endosurgery, Cook Endoscopy, Aspire Bariatrics, GI Dynamics, Olympus, NinePoint Medical, Merit Medical, and MI Technologies. He is a consultant for Boston Scientific, Xlumena, Concordia Laboratories, and Mauna Kea Technologies. None of the other authors has any conflicts of interest to report.

\section{Institutions}

${ }^{1}$ Division of Gastroenterology and Hepatology, Weill Cornell Medical College, New York, New York, USA

${ }^{2}$ Gastroenterology Department, Hospital Universitario Rio Hortega, Valladolid, Spain

3 Paul May and Frank Stein Interventional Endoscopy Center, California Pacific Medical Center, San Francisco, California, USA

${ }^{4}$ Division of Gastroenterology and Hepatology, UNC School of Medicine, Chapel Hill, North Carolina, USA

\section{References}

1 Medina-Franco H, Abarca-Pérez L, España-Gómez $N$ et al. Morbidityassociated factors after gastrojejunostomy for malignant gastric outlet obstruction. Am Surg 2007; 73: 871-875

2 Khashab M, Alawad AS, Shin EJ et al. Enteral stenting versus gastrojejunostomy for palliation of malignant gastric outlet obstruction. Surg Endosc 2013; 27: 2068 - 2075

3 van Hooft JE, Uitdehaag MJ, Bruno MJ et al. Efficacy and safety of the new WallFlex enteral stent in palliative treatment of malignant gastric outlet obstruction (DUOFLEX study): a prospective multicenter study. Gastrointest Endosc 2009; 69: 1059-1066
4 Phillips MS, Gosain S, Bonatti $H$ et al. Enteral stents for malignancy: a report of 46 consecutive cases over 10 years, with critical review of complications. J Gastrointest Surg 2008; 12: 2045 - 2050

5 Binmoeller KF, Shah JN. Endoscopic ultrasound-guided gastroenterostomy using novel tools designed for transluminal therapy: a porcine study. Endoscopy 2012; 44: 499-503

6 Itoi $T$, Ishii $K$, Tanaka $R$ et al. Current status and perspective of endoscopic ultrasonography-guided gastrojejunostomy: endoscopic ultrasonography-guided double-balloon-occluded gastrojejunostomy (with videos). J Hepatobiliary Pancreat Sci 2015; 22: 3-11

7 Luo H, Pan Y, Min L et al. Transgastric endoscopic gastroenterostomy using a partially covered occluder: a canine feasibility study. Endoscopy 2012; 44: $493-498$

8 Vanbiervliet $G$, Bonin EA, Garcès $R$ et al. Gastrojejunal anastomosis using a tissue-apposing stent: a safety and feasibility study in live pigs. Endoscopy 2014; 46: $871-877$

9 Barthet M, Binmoeller KF, Vanbiervliet $G$ et al. Natural orifice transluminal endoscopic surgery gastroenterostomy with a biflanged lumenapposing stent: first clinical experience (with videos). Gastrointest Endosc 2015; 81: 215-218

10 Tyberg A, Kumta N, Karia K et al. EUS-guided gastrojejunostomy after failed enteral stenting. Gastrointest Endosc 2015; 81: 1011 - 1012

11 Itoi $T$, Tsuchiya T, Tonozuka $R$ et al. Novel EUS-guided double-balloonoccluded gastrojejunostomy bypass. Gastrointest Endosc 2015: [Epub]

12 Tyberg A, Zerbo S, Barthet $M$ et al. A novel technique for salvaging a dislodged lumen-apposing metal stent during creation of an endoscopic gastrojejunostomy. Gastrointest Endosc 2015: [Epub]

13 Itoi T, Ishii K, Ikeuchi $N$ et al. Prospective evaluation of endoscopic ultrasonography-guided double-balloon-occluded gastrojejunostomy bypass (EPASS) for malignant gastric outlet obstruction. Gut 2015 [Epub]

14 Khashab MA, Kumbhari V, Grimm IS et al. EUS-guided gastroenterostomy: the first U.S. clinical experience (with video). Gastrointest Endosc In press 2015: DOI 10.1016/j.gie.2015.06.017

15 De la Serna-Higuera C, Pérez-Miranda M, Gil-Simón P et al. EUS-guided transenteric gallbladder drainage with a new fistula-forming, lumenapposing metal stent. Gastrointest Endosc 2013; 77: 303 - 308 